\title{
ARTICLE
}

Epidemiology and Population Health

\section{Macronutrient intake during pregnancy in women with a history of obesity or gestational diabetes and offspring adiposity at 5 years of age}

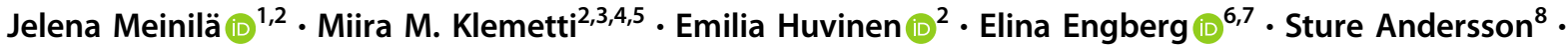 \\ Beata Stach-Lempinen ${ }^{3} \cdot$ Saila Koivusalo $^{2}$
}

Received: 13 April 2020 / Revised: 8 December 2020 / Accepted: 20 January 2021 / Published online: 8 February 2021

(c) The Author(s) 2021. This article is published with open access

\begin{abstract}
Background/objectives The impact of maternal macronutrient intake during pregnancy on offspring childhood adiposity is unclear. We assessed the associations between maternal macronutrient intake during and after pregnancy with offspring adiposity at 5 years of age. Additionally, we investigated whether gestational diabetes (GDM), BMI, or breastfeeding modified these associations.

Subjects/methods Altogether, 301 mother-child dyads with maternal prepregnancy BMI $\geq 30$ and/or previous GDM participated in the Finnish Gestational Diabetes Prevention Study (RADIEL) and its 5 years follow-up. Macronutrient intakes (E\%) were calculated from 3-day food records collected at 5-18 weeks' gestation, in the third trimester, and at 12 months and 5 years after pregnancy. Offspring body fat mass (BFM) and fat percentage (BF\%) at 5 years were measured by bioimpedance. Statistical analyses were multivariate linear regression.

Results Mean (SD) prepregnancy BMI was 33(4) kg/m². GDM was diagnosed in 47\%. In normoglycemic women, higher first half of pregnancy n-3 PUFA intake was associated with lower offspring BFM $(\mathrm{g})(\beta-0.90 ; 95 \% \mathrm{CI}-1.62,-0.18)$ and $\mathrm{BF} \%(\beta-3.45 ; 95 \% \mathrm{CI}-6.17,-0.72)$. In women with GDM, higher first half of pregnancy n-3 PUFA intake was associated with higher offspring BFM $(\beta 0.94 ; 95 \%$ CI $0.14,1.75)$ and BF\% $(\beta 3.21 ; 95 \%$ CI 0.43 , 5.99). Higher SFA intake in the third trimester and cumulative intake across pregnancy (mean of the first half and late pregnancy) was associated with higher BFM and BF\% (across pregnancy: $\beta 0.12 ; 95 \%$ CI $0.03,0.20$ and $\beta 0.44 ; 95 \%$ CI $0.15,0.73$, respectively). Higher carbohydrate intake across pregnancy was associated with lower BFM $(\beta-0.044 ; 95 \% \mathrm{CI}-0.086,-0.003)$, and borderline associated with $\mathrm{BF} \%(\beta-0.15 ; 95 \% \mathrm{CI}-0.31,0.00)$.

Conclusions The macronutrient composition of maternal diet during pregnancy is associated with offspring BFM and BF\% at 5 years. GDM modifies the association between prenatal n-3 PUFA intake and offspring anthropometrics.
\end{abstract}

Supplementary information The online version contains supplementary material available at https://doi.org/10.1038/s41366021-00762-0.

Jelena Meinilä

jelena.meinila@helsinki.fi

1 Department of Food and Nutrition, University of Helsinki, Helsinki, Finland

2 Department of Obstetrics and Gynecology, University of Helsinki and Helsinki University Hospital, Helsinki, Finland

3 Department of Obstetrics and Gynecology, South Karelia Central Hospital, Lappeenranta, Finland

\section{Introduction}

Obesity is rapidly increasing in children and adolescents worldwide. In 2019, 38 million children younger than 5 years were overweight or obese [1]. In accordance with

4 Department of Medical and Clinical Genetics, University of Helsinki and Helsinki University Hospital, Helsinki, Finland

5 Lunenfeld-Tanenbaum Research Institute, Mount Sinai Hospital, Toronto, ON, Canada

6 Folkhälsan Research Center, Helsinki, Finland

7 Faculty of Medicine, University of Helsinki, Helsinki, Finland

8 Children's Hospital, Pediatric Research Center, University of Helsinki and Helsinki University Hospital, Helsinki, Finland 
global trends, over $25 \%$ of Finnish preschool-aged boys and over $15 \%$ of girls are currently overweight or obese [2]. Child obesity is associated with reduced quality of life in childhood [3], cardiometabolic morbidity in later life [4], and premature death [5]. Therefore, identification and targeting of modifiable risk factors for childhood obesity should be a public health priority.

Mounting evidence indicates that maternal health behaviors such as physical activity, smoking, and diet during pregnancy are associated with long-term health outcomes in the offspring [6-8]. Data from animal and human studies suggest that, in addition to maternal gestational diabetes (GDM) and obesity [9-11], in utero exposure to poor quality diet (e.g., under- or over-nutrition) predisposes the offspring to excess adiposity $[12,13]$. Recent findings also suggest that the macronutrient composition of maternal diet during pregnancy is associated with indicators of newborn adiposity (body mass index (BMI), waist circumference, body fat mass (BFM), and body fat percentage (BF\%)) $[8,14,15]$. However, only a few studies have examined the associations between maternal macronutrient intake and offspring adiposity at later ages, and analyses extending beyond maternal polyunsaturated fatty acid (PUFA) intake are particularly scarce [8, 16-19]. Some of the available studies have relied only on indirect measures of adiposity, such as weight [16] or BMI [17, 19]. Furthermore, only one study has attempted to differentiate between the effects of maternal nutrient intake during pregnancy and the child's own nutrient intake in early childhood by analyzing maternal macronutrient intake after pregnancy as an indicator of the food environment of the offspring [16]. None of the existing studies have taken into account the child's own macronutrient intake. Moreover, whether the impact of maternal macronutrient intake on the offspring body composition varies across gestation remains unclear.

The aim of this study was to examine the associations between the macronutrient composition of maternal diet during the first half of and late pregnancy and offspring anthropometric indicators at 5 years of age (age- and sexadjusted BMI (ISO-BMI), waist-to-height ratio (WHtR), $\mathrm{BFM}$, and $\mathrm{BF} \%$ ). To ensure that none of the observed associations between macronutrient intakes during pregnancy and offspring anthropometric indicators result from confounding by the child's own food environment during childhood, we also studied the associations between maternal macronutrient intakes at 12 months and 5 years after pregnancy and offspring adiposity.

\section{Subjects and methods}

All background and pregnancy data were originally collected during the RADIEL study, a multi-center randomized controlled intervention trial aimed at prevention of GDM with diet and physical activity counseling [20]. The trial involved a total of 787 women at high risk for GDM who delivered in the Helsinki metropolitan area or in Lappeenranta, Finland, during 2008-2011. The recruited women were obese $\left(B M I \geq 30 \mathrm{~kg} / \mathrm{m}^{2}\right)$ and/or had been diagnosed with GDM in a previous pregnancy. At enrollment, the women were either $\leq 20$ weeks pregnant or planning pregnancy. Exclusion criteria were age $<18$ years, preexisting type 1 or 2 diabetes mellitus, physical disability, multiple pregnancy, severe psychiatric disorders, current substance abuse, substantial communication difficulties and using medication affecting glucose metabolism (e.g., corticosteroids, metformin, antidepressants with potential effects on glucose homeostasis).

Five years after the trial, during 2013-2017, the participants and their offspring were invited for follow-up examinations. The study population for the present study was formed as depicted in Fig. 1. The number of mother-child pairs who participated in the follow-up was 332; 301 provided both a completed maternal 3-day food record at least once during pregnancy (in the first half of pregnancy 5-18 weeks' gestation or in the third trimester) and the offspring's weight and height at 5 years. At least one maternal food record and the offspring's BFM and BF $\%$ measurements at 5 years of age were available from 274 pairs. Maternal 3-day food records from both the first half of pregnancy and third trimester of pregnancy and offspring's weight and height at 5 years of age were available from 230 mother-child pairs, with offspring BFM and BF\% at 5 years additionally available from 201 of these pairs. In the present study, the intervention and control groups were combined and treated as a cohort and observational analyses were performed. The study was conducted according to the guidelines laid down in the Helsinki Declaration of 1975 as revised in 1983. All procedures involving human subjects were approved by the Ethics Committee of the Department of Obstetrics and Gynecology of Helsinki and Uusimaa Hospital District. Written informed consent was obtained from all subjects in the main trial and in the 5-year follow-up. In the follow-up study, informed consent on behalf of the child was obtained from the parents.

\section{Nutrient intake of the mother and child}

Maternal macronutrient intake during pregnancy was calculated from two 3-day food records collected in the first half of pregnancy (gestational weeks 5-18, mean 13 \pm 2 ) and in the third trimester of pregnancy (mean gestational weeks $35 \pm 1$ ), as well as at 12 months and 5 years after delivery. At the initial trial enrollment visit in the first half of pregnancy, mothers received written instructions 


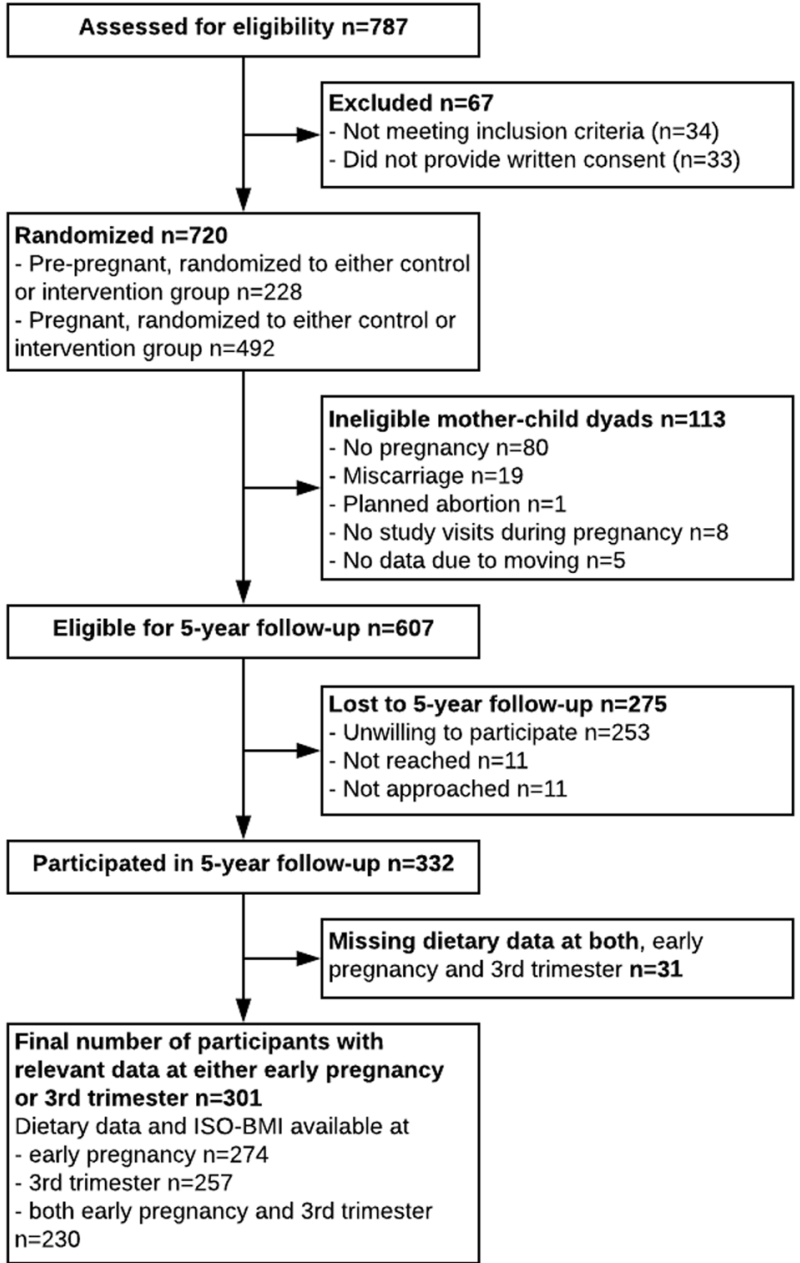

Fig. 1 Formation of the study population. The first half of pregnancy contains gestational weeks from 5 to 18. ISO-BMI represents age- and sex-standardized BMI converted to adult scale and was calculated according to Saari et al. [23].

for completing food records on three consecutive days (two weekdays and a weekend day) immediately or as soon as possible after each meal. They were asked to report food and beverage labels and brand names as accurately as possible, and record amounts as household measures (e.g., tablespoon, plate, scoop) or in weight units, if available. Identical instructions were given for completing 3-day food records in the third trimester of pregnancy and at 12 months and 5 years after delivery.

Similarly, the offspring's macronutrient intake at 5 years of age was calculated from 3-day food records completed by their caretaker (usually mother or father) with similar accuracy as instructed for maternal food records. If the 3-day recording period included daycare time, the childcare providers were asked to complete a separate food record with identical instructions. Recipes and ingredients used in daycare meals were collected from the food suppliers or the daycare kitchen.
Trained nutritionists processed and entered the food record data into a nutritional calculation software, AivoDiet (versions 2.0.1.5 and 2.2.0.1, Aivo Finland Oy, Turku, Finland). A table of usual portion sizes helped in converting household measures and volumes into grams [21]. The food composition database was provided by the Finnish National Institute for Health and Welfare (www.fineli.fi). The nutrient values in the database rely mostly on Finnish studies, in addition to data obtained from Finnish food industries and international food composition tables. The database includes standard recipes that are based on contemporary Finnish cookbooks. If a food or recipe reported in the food record was missing, a new recipe was created based on the food record.

\section{Collection of maternal background information}

At the initial trial enrollment and 5-year follow-up visits, the mothers completed a background questionnaire on socio-demographic factors, earlier pregnancies and deliveries, and health-related behaviors during the preceding 6 months. Information on GDM was based on medical records and physician confirmation, except for two participants for whom this was unavailable. Prepregnancy BMI was calculated from weight and height measured at the first study visit (in those recruited before pregnancy) or was self-reported (in those recruited in the first half of pregnancy). Duration of breastfeeding $(<4$ months/ $\geq 4$ months) was collected from the records of the child health centres which monitored the children's health, growth, and development.

\section{Collection of offspring background data and anthropometric measurements}

Data on offspring sex, birthweight, and gestational age at birth were collected from hospital records. Relative birthweight was calculated using Finnish fetal growth curves adjusted for sex and gestational age [22]. Measurements from the child participants were taken in the research centers of the Hospital District of Helsinki and Uusimaa or in South Karelia Central Hospital by trained study nurses. Height was measured with feet shoeless, flat, together, and against the wall. Weight was measured with a digital scale in light clothing. Waist circumference was measured at midway between the lowest ribs and the iliac crest. Measures of height, weight, and waist were rounded to one decimal. Offspring BFM and BF\% at 5 years were measured during the 5-year visit using Bioelectrical Impedance Analysis (InBody 720 eight-polar tactile electrode system (Biospace Co., Ltd, Seoul, South Korea)). Offspring sexspecific BMI-for-age at 5 years was calculated according to the Finnish growth curves and was converted to adult scale 
(ISO-BMI) according to Saari et al. [23]. Offspring WHtR was calculated as waist circumference $(\mathrm{cm})$ divided by height (m).

\section{Statistical methods}

Descriptive characteristics are presented as means (SD) or frequencies (\%). Macronutrient intakes in the first half of pregnancy and in the third trimester of pregnancy were analyzed both separately and combined (mean of the two measures) to represent cumulative intake across pregnancy. Associations between maternal macronutrient intakes and offspring $z$-birthweight, and ISO-BMI, WHtR, $\mathrm{BFM}$, and $\mathrm{BF} \%$ at 5 years were analyzed by linear regression analysis. Model 1 included mother's energy intake as a covariate. Model 2 additionally included potential confounding factors such as maternal age during pregnancy, educational attainment (years), smoking in the first half of pregnancy (yes/no), GDM status (yes/no), intervention allocation (control/intervention during the main trial), maternal prepregnancy BMI, offspring sex, offspring age at 5-year follow-up visit, offspring's zbirthweight, and offspring's intake of the given nutrient (E\%) at 5 years of age. Additionally, we constructed a model without the variables that could potentially be involved in the causal pathways between maternal macronutrient intake and offspring anthropometric measures: GDM status, intervention allocation, maternal prepregnancy BMI, offspring z-birthweight, and offspring's intake of the index nutrient at 5 years of age. The results of this additional model are not presented since they did not differ substantially from those obtained with Model 2. Information on maternal leisure-time physical activity (LTPA) and duration of breastfeeding, potential confounders in the association between maternal diet and offspring anthropometric indicators, were missing from several participants (LTPA: 34 mothers with only first half of pregnancy food records, 17 mothers with only third trimester food records, and 42 mothers with two food records during pregnancy; breastfeeding: 28 mothers with only first half of pregnancy food records, 7 mothers with only third trimester food records, and 23 mothers with two food records during pregnancy). Therefore, these variables were omitted from the main analyses. However, in sensitivity analyses, LTPA and duration of breastfeeding were added separately as covariates, in addition to the variables of Model 2. We also tested nutrient interaction with variables GDM, duration of breastfeeding, and prepregnancy BMI on offspring anthropometric indicators. Each interaction was tested in a separate analysis by including in the models both the interaction term $([\mathrm{GDM} \times$ nutrient intake $(\mathrm{E} \%)] /[$ duration of breastfeeding $\times$ nutrient intake $(\mathrm{E} \%)] /[$ prepregnancy $\mathrm{BMI} \times$ nutrient intake (E\%)]) and its components as independent variables, together with the other covariates of Model 2. This was performed for all studied nutrients in all timepoints (first half of pregnancy, third trimester, and 12 months and 5 years after pregnancy). If an interaction was found, the analyses were performed in subgroups according to the variable with which the interaction was identified.

If an association between maternal intake of a macronutrient and offspring ISO-BMI, WHtR, BFM, or BF\% was found, additional analyses were performed to assess whether the observed association was due to intake during pregnancy or due to maintained nutrient intake after pregnancy (which could reflect the food environment of the offspring). The nutrient intakes of Model 2 were replaced with maternal intakes at 12 months or 5 years after delivery, and the offspring's own intakes of specific nutrients were excluded from the model.

We also tested quadratic associations between macronutrients and BFM and BF\% by adding a squared macronutrient intake in addition to macronutrient intake in Model 2. These were tested in the same groups as the main analyses (i.e., in the total cohort, or in subgroups, if interactions were observed).

In case of violation of the assumption of normality, a bootstrap method was used. Equality of variances were tested by Levene's test. Statistical software Stata, version 13.1 (Stata Corporation, College Station, TX, USA) was used in all analyses.

\section{Results}

\section{Maternal characteristics}

Maternal background characteristics are shown in Table 1. The mean (SD) maternal age was 33 (4) years and the mean prepregnancy BMI was $31(6) \mathrm{kg} / \mathrm{m}^{2}$. Almost half of the mothers $(n=141 ; 47 \%)$ had GDM during the index pregnancy. The mean (SD) age of the offspring at followup was 5.0 years (0.5) and $138(46 \%)$ were girls. The mean (SD) BF\% of girls was 19 (6) and that of boys 15 (6). The mean (SD) ISO-BMI for both the girls and boys was 23 (4). Table 2 displays the mean maternal macronutrient intakes and time spent on LTPA during pregnancy, separately in the first half of pregnancy and third trimester, and combined to reflect total exposure across pregnancy.

\section{Maternal macronutrient intake and offspring relative birthweight}

None of the maternal macronutrient intakes were associated with offspring's relative birthweight (Supplementary Table 2). 
Table 1 Maternal and offspring characteristics and offspring anthropometric indicators at 5 years of age in mother-child dyads with maternal 3-day food records completed in both first half of pregnancy (5-18 weeks' gestation) and third trimester, and in those with food records completed only once during pregnancy.

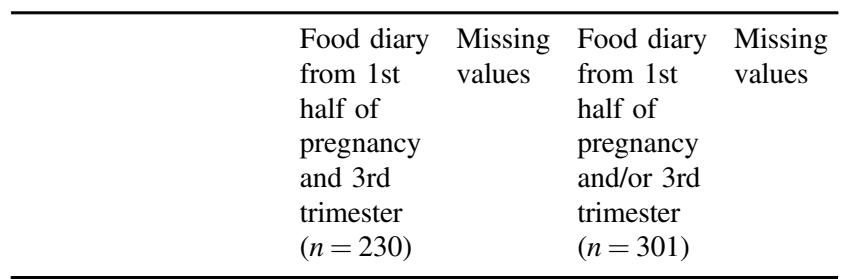

Mother

\begin{tabular}{|c|c|c|c|c|c|c|}
\hline Age, year, mean (SD) & 32.9 & $(4.4)$ & 0 & 33.0 & $(4.5)$ & 0 \\
\hline $\begin{array}{l}\text { BMI at } 1 \mathrm{st} \text { half of } \\
\text { pregnancy, } \mathrm{kg} / \mathrm{m}^{2}, \\
\text { mean }(\mathrm{SD})\end{array}$ & 30.9 & $(5.6)$ & 0 & 31.1 & $(5.7)$ & 0 \\
\hline $\begin{array}{l}\text { Educational } \\
\text { attainment, year, } \\
\text { mean (SD) }\end{array}$ & 14.7 & $(2.0)$ & 0 & 14.6 & $(2.0)$ & 1 \\
\hline $\begin{array}{l}\text { Current smokers (in } \\
1 \text { st trimester), } n(\%)\end{array}$ & 8 & (4) & 1 & 12 & (4) & 1 \\
\hline $\begin{array}{l}\text { Gestational age at } \\
\text { early pregnancy food } \\
\text { recording, mean (SD) }\end{array}$ & 13.1 & $(1.8)$ & 1 & 13.1 & $(1.8)$ & 1 \\
\hline $\begin{array}{l}\text { Gestational age at } \\
\text { 3rd trimester food } \\
\text { recording, mean (SD) }\end{array}$ & 34.9 & $(1.1)$ & 2 & 34.9 & $(1.2)$ & 4 \\
\hline $\begin{array}{l}\text { Gestational diabetes, } \\
n(\%)\end{array}$ & 101 & (44) & 0 & 141 & (47) & 0 \\
\hline $\begin{array}{l}\text { Gestational age at } \\
\text { delivery, week, } \\
\text { mean (SD) }\end{array}$ & 39.9 & $(1.4)$ & 0 & 39.9 & $(1.5)$ & 0 \\
\hline $\begin{array}{l}\text { Breastfeeding, } \\
\text { months, mean (SD) }\end{array}$ & 4.2 & $(2.9)$ & 17 & 4.2 & $(2.9)$ & 21 \\
\hline \multicolumn{7}{|l|}{ Child } \\
\hline Boys, $n(\%)$ & 122 & (53) & 0 & 163 & (54) & 0 \\
\hline Girls & 108 & (47) & 0 & 138 & (46) & 0 \\
\hline \multicolumn{7}{|l|}{ At birth } \\
\hline $\begin{array}{l}\text { Birthweight, g, } \\
\text { mean (SD) }\end{array}$ & 3632 & (494) & 0 & 3664 & $(503)$ & 0 \\
\hline $\begin{array}{l}z \text {-birthweight, } \\
\text { mean (SD) }\end{array}$ & 0.1 & $(0.9)$ & 0 & 0.2 & $(1.0)$ & 0 \\
\hline \multicolumn{7}{|l|}{ At 5-year follow-up } \\
\hline Age, year, mean (SD) & 5.0 & $(0.5)$ & 0 & 5.0 & $(0.5)$ & 0 \\
\hline \multicolumn{7}{|l|}{ Waist } \\
\hline Boys & 54.3 & $(3.5)$ & 6 & 54.6 & $(3.9)$ & 10 \\
\hline Girls & 54.9 & $(5.0)$ & 5 & 54.9 & $(4.9)$ & 9 \\
\hline \multicolumn{7}{|c|}{ Waist-height ratio, mean (SD) } \\
\hline Boys & 48.7 & $(2.6)$ & 6 & 48.8 & $(2.9)$ & 10 \\
\hline Girls & 49.8 & $(3.9)$ & 5 & 49.7 & $(3.9)$ & 9 \\
\hline \multicolumn{7}{|l|}{ BMI, mean (SD) } \\
\hline Boys & 16.0 & $(1.3)$ & 0 & 16.1 & $(1.4)$ & 0 \\
\hline Girls & 16.4 & $(1.8)$ & 0 & 16.4 & $(1.7)$ & 0 \\
\hline
\end{tabular}

Table 1 (continued)

\begin{tabular}{|c|c|c|c|c|}
\hline & $\begin{array}{l}\text { Food diary } \\
\text { from 1st } \\
\text { half of } \\
\text { pregnancy } \\
\text { and 3rd } \\
\text { trimester } \\
(n=230)\end{array}$ & $\begin{array}{l}\text { Missing } \\
\text { values }\end{array}$ & $\begin{array}{l}\text { Food diary } \\
\text { from } 1 \text { st } \\
\text { half of } \\
\text { pregnancy } \\
\text { and/or 3rd } \\
\text { trimester } \\
(n=301)\end{array}$ & $\begin{array}{l}\text { Missing } \\
\text { values }\end{array}$ \\
\hline \multicolumn{5}{|c|}{ ISO-BMI, mean (SD) } \\
\hline Boys & $22.9 \quad(3.6)$ & 0 & $23.1 \quad(4.0)$ & 0 \\
\hline Girls & $22.5 \quad(4.1)$ & 0 & $22.6 \quad(4.0)$ & 0 \\
\hline \multicolumn{5}{|c|}{ Fat mass, kg, mean (SD) } \\
\hline Boys & $(1.3)$ & 13 & $(1.5)$ & 19 \\
\hline Girls & $(2.0)$ & 16 & $(4.0)$ & 16 \\
\hline \multicolumn{5}{|c|}{ Fat as $\%$ from total bodyweight, mean (SD) } \\
\hline Boys & $14.6 \quad(5.4)$ & 13 & $14.9 \quad(5.6)$ & 19 \\
\hline Girls & $19.2(6.1)$ & 29 & $19.1 \quad(6.1)$ & 16 \\
\hline
\end{tabular}

$z$-birthweight represents sex- and gestational age-adjusted birthweight [22]. ISO-BMI represents age- and sex-standardized BMI converted to adult scale [23].

\section{Maternal macronutrient intake and offspring ISO- BMI and waist-to-height ratio (WHtR)}

GDM, BMI, or breastfeeding did not modify any associations between macronutrients and ISO-BMI and WHtR (all $p$ for interaction $>0.05$ ). Higher third trimester saturated fatty acid (SFA) intake was associated with higher ISO-BMI (Model 2), but the first half of pregnancy intake or combined intake across pregnancy were not (Table 3). No associations between maternal macronutrient intakes (in the first half of pregnancy, third trimester, or combined) and WHtR were detected.

\section{Maternal macronutrient intake and offspring BFM and $\mathrm{BF} \%$}

GDM status modified the association between first half of pregnancy n-3 PUFA intake and offspring BFM ( $p$ for interaction <0.001) and $\mathrm{BF} \%(p$ for interaction $=0.001)$ at 5 years of age. In women with normal glucose metabolism during the index pregnancy, higher first half of pregnancy intake of n-3 PUFA was associated with lower offspring $\mathrm{BFM}$ and $\mathrm{BF} \%$ at 5 years of age (Table 4). In women with GDM, however, higher first half of pregnancy intake of n-3 PUFA was associated with higher offspring BFM and BF\% at 5 years of age. There were no differences in first half of pregnancy n-3 PUFA intakes between women with normal glucose tolerance and women with GDM (1.75 (0.39) vs. $1.8(0.40), p=0.70)$.

The association between maternal first half of pregnancy n-3 PUFA intake and offspring BF\% pointed toward the 
Table 2 Maternal macronutrient intakes and duration of leisuretime physical activity per week in first half of pregnancy, in the third trimester of pregnancy, and as an average of the two timepoints.

\begin{tabular}{|c|c|c|c|c|c|c|c|c|c|c|}
\hline & \multicolumn{3}{|c|}{$\begin{array}{l}\text { 1st half of } \\
\text { pregnancy } \\
\text { (gestational weeks } \\
5-18 \text { ) }\end{array}$} & \multicolumn{3}{|c|}{$\begin{array}{l}\text { 3rd trimester of } \\
\text { pregnancy }\end{array}$} & \multirow[b]{2}{*}{$p$} & \multicolumn{3}{|c|}{$\begin{array}{l}\text { Combined 1st half } \\
\text { and 3rd trimester }\end{array}$} \\
\hline & $n$ & Mean & sd & $n$ & Mean & sd & & $n$ & Mean & sd \\
\hline Nutrition & 274 & & & 257 & & & & 230 & & \\
\hline Energy intake, $\mathrm{kcal} / \mathrm{d}$ & & 1900 & 420 & & 1950 & 440 & 0.50 & & 1930 & 380 \\
\hline Carbohydrates, E\% & & 44.8 & 6.0 & & 43.5 & 7.0 & 0.01 & & 44.0 & 5.5 \\
\hline Sucrose, E\% & & 8.5 & 3.8 & & 8.5 & 4.5 & 0.31 & & 8.4 & 3.3 \\
\hline Dietary fiber, g/1000 kcal & & 12.6 & 3.8 & & 12.5 & 3.9 & 0.77 & & 12.5 & 3.3 \\
\hline Total fat, E\% & & 33.6 & 5.7 & & 34.8 & 6.8 & 0.02 & & 34.3 & 5.3 \\
\hline SFA, E\% & & 12.3 & 2.9 & & 12.8 & 3.5 & 0.10 & & 12.6 & 2.7 \\
\hline MUFA, E\% & & 11.6 & 2.3 & & 11.9 & 2.6 & 0.04 & & 11.8 & 2.0 \\
\hline n-3 PUFA, E\% & & 1.8 & 0.4 & & 1.8 & 0.5 & 0.50 & & 1.8 & 0.3 \\
\hline n-6 PUFA, E\% & & 4.5 & 1.1 & & 4.7 & 1.4 & 0.03 & & 4.6 & 1.0 \\
\hline Protein, E\% & & 18.1 & 3.1 & & 18.3 & 3.1 & 0.21 & & 18.2 & 2.5 \\
\hline Duration of LTPA per week (min) & 240 & 105 & 110 & 240 & 81 & 107 & 0.12 & 191 & 94 & 88 \\
\hline
\end{tabular}

$E \%$ percentage of energy from total energy intake, SFA saturated fatty acids, MUFA monounsaturated fatty acids, PUFA polyunsaturated fatty acids, LTPA leisure-time physical activity. same direction with and without adjustment for LTPA, both in women with normal glucose tolerance and in those with GDM. However, this association was not significant in those with GDM (Supplementary Table 1).

GDM did not modify the associations between third trimester maternal n-3 PUFA intake and BFM or BF\%, and, in the total cohort, these associations were not statistically significant (Table 4). GDM did not modify any other associations between macronutrients and BFM and $\mathrm{BF} \%$. Prepregnancy BMI and breastfeeding did not modify any associations between macronutrients and BFM and BF\% (all $p$ for interaction $>0.05$ ).

Both higher third trimester SFA intake and higher combined SFA intake across pregnancy were associated with higher offspring BFM and BF\% (in Models 1 and 2, Table 5).

Higher carbohydrate (CHO) intake across pregnancy was associated with lower offspring BFM and borderline associated with lower BF\% (in Model 2). No other associations between maternal macronutrient intakes and offspring BFM and $\mathrm{BF} \%$ were observed.

\section{Sensitivity analyses}

Because maternal n-3 PUFA, SFA, and CHO intakes during pregnancy were associated with offspring ISO-BMI, BFM, and $\mathrm{BF} \%$, we also examined the associations between maternal n-3 PUFA, SFA, and CHO intakes at 12 months and 5 years after delivery and offspring anthropometric indicators. However, no associations with offspring ISOBMI, BFM, or BF\% at 12 months or 5 years were detected (Supplementary Table 3). No quadratic associations between maternal macronutrient intakes and offspring BFM or $\mathrm{BF} \%$ were found.

\section{Discussion}

We found that GDM modified the associations between maternal intake of n-3 PUFAs in the first half of pregnancy and offspring BFM and BF\% at 5 years of age. Maternal intake of SFA during pregnancy was positively associated with offspring ISO-BMI, BFM, and $\mathrm{BF} \%$. In contrast, maternal $\mathrm{CHO}$ intake across pregnancy was negatively associated with offspring BFM and BF\%.

Only a few previous human studies have examined associations between maternal macronutrient intakes during pregnancy and offspring adiposity after infancy [8, 16-19]. Among the strengths of our study are the collection of detailed maternal nutrient intake data at four timepoints, during and after pregnancy, and the inclusion of data on the offspring's own nutrient intake. These enabled us to assess the role of timing of maternal macronutrient intake during pregnancy and the food environment of the offspring after birth in the development of offspring adiposity. No associations between maternal macronutrient intakes after pregnancy and offspring anthropometric indictors were found, and our analyses were adjusted for the child's own nutrient intake at 5 years. Hence, it is unlikely that our findings result from confounding by maternal or child diet composition after delivery.

Compared to the results of the present study, previous cohort studies have reported both congruent $[17,18]$ and discrepant [19] findings on the association of maternal n-3 


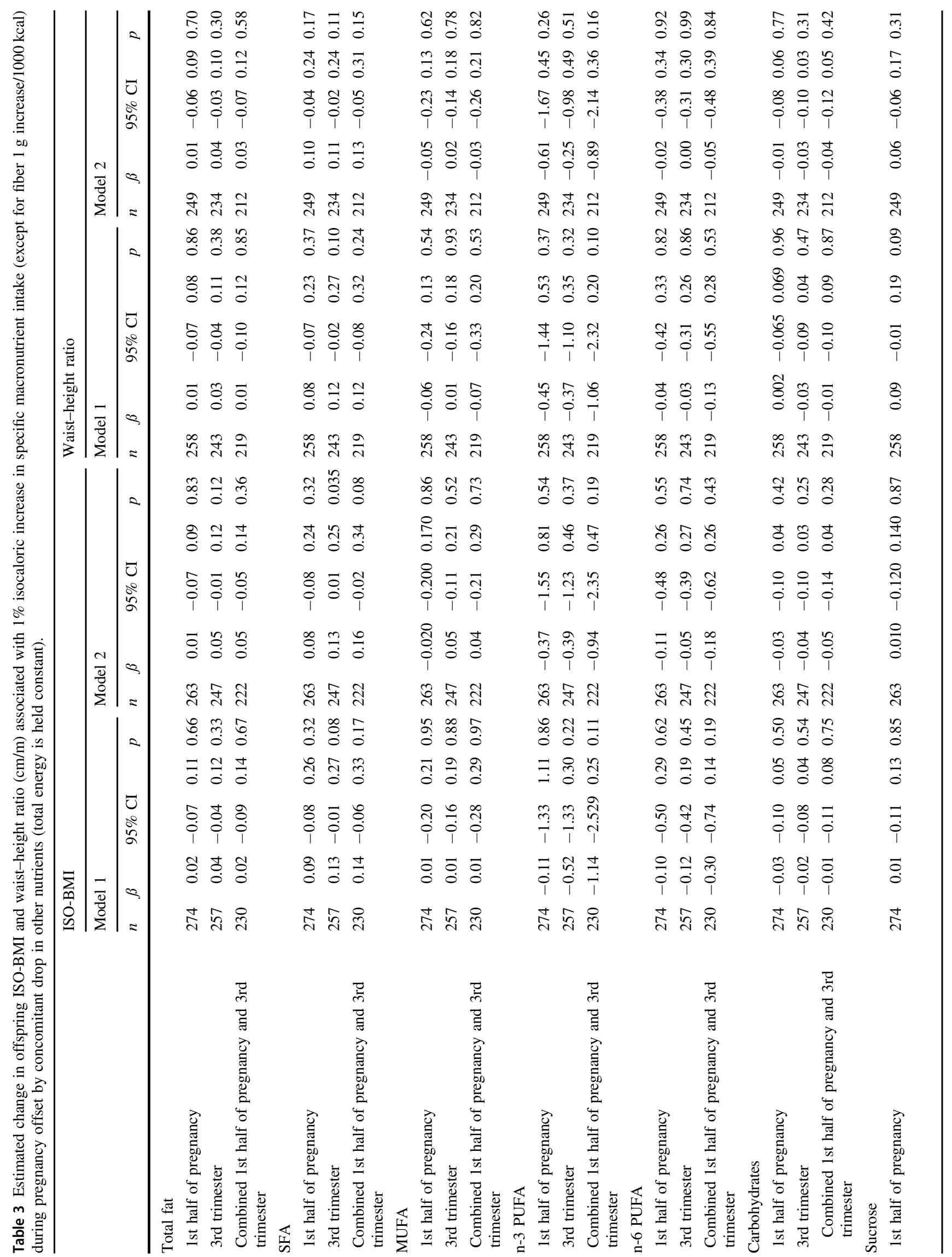




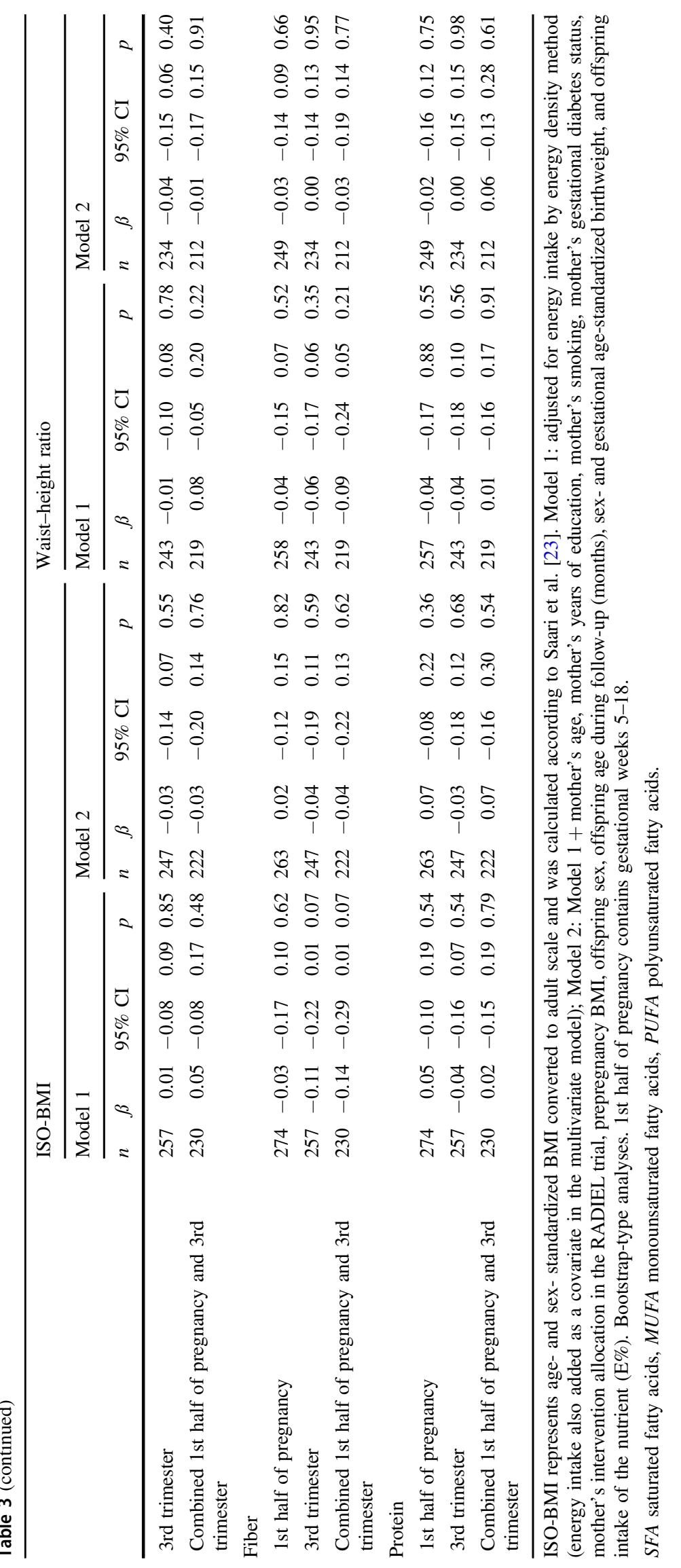


PUFA intake during pregnancy and offspring adiposity. Differences in the impact of maternal n-3 PUFA intakes in healthy women vs. women with GDM in the first half of pregnancy on offspring body composition could explain some of these inconsistencies, as earlier studies have examined average nutrient intakes across the entire pregnancy. More recently, Brei et al. [8] showed that higher late pregnancy n-3 PUFA intake is associated with lower birthweight and newborn fat mass in the offspring, although these associations did not persist until 1 year of age. Donahue et al. $(n=1120)$, however, found an association between higher maternal n-3 PUFA intake and lower sum of subscapular and tricep skinfold thicknesses in children at 3 years of age [18], conforming to our results in women without GDM. n-3 PUFAs block adipose tissue development, leading to lower fat mass [24], which could possibly contribute to the association of late pregnancy maternal PUFA intake with reduced offspring adiposity, although this finding did not reach significance in our study. In a mouse model, increased maternal n-3 fatty acid intake (reduced n-6/n-3 PUFA ratio) was shown to lead to lower body fat and traits indicative of an obesity-resistant phenotype in the offspring [24]. However, although cohort studies have repeatedly found inverse associations between maternal n-3 PUFA intake during pregnancy and offspring adiposity $[18,25]$, these have not been replicated in clinical trials testing n-3 PUFA supplementation in pregnant women [26].

Long-chain PUFAs are essential for normal fetal growth and development. Since placental and fetal capacity to synthesize them is low, the fetus depends on the maternal supply of PUFAs [27]. Earlier studies have suggested derangements in the placental transfer and/or feto-placental metabolism of PUFAs in late pregnancy in women with GDM or obesity [28-32], which could contribute to the lack of benefit on offspring adiposity from increased late pregnancy n-3 PUFA intake in our study population. Similar data on the first half of pregnancy is lacking and the flux of lipids to the fetus is considerably lower at $<20$ weeks gestation than at term [28]. However, GDM is characterized by various maternal metabolic abnormalities (e.g., elevated glucose and insulin) already in early pregnancy [33, 34], and early changes in placental metabolism and growth in response to disturbed maternal metabolism are increasingly recognized as potential factors in the pathogenesis of fetal adiposity [35-38]. Considering our observation of increased offspring adiposity in women with GDM with high first half of pregnancy n-3 PUFA intake, it is possible that placental n-3 PUFA metabolism is altered in GDM already in the first half of pregnancy.

Current evidence regarding the impact of maternal SFA intake on offspring body composition is inconsistent. In a large Finnish birth cohort study by Hakola et al. $(n=3807)$ 


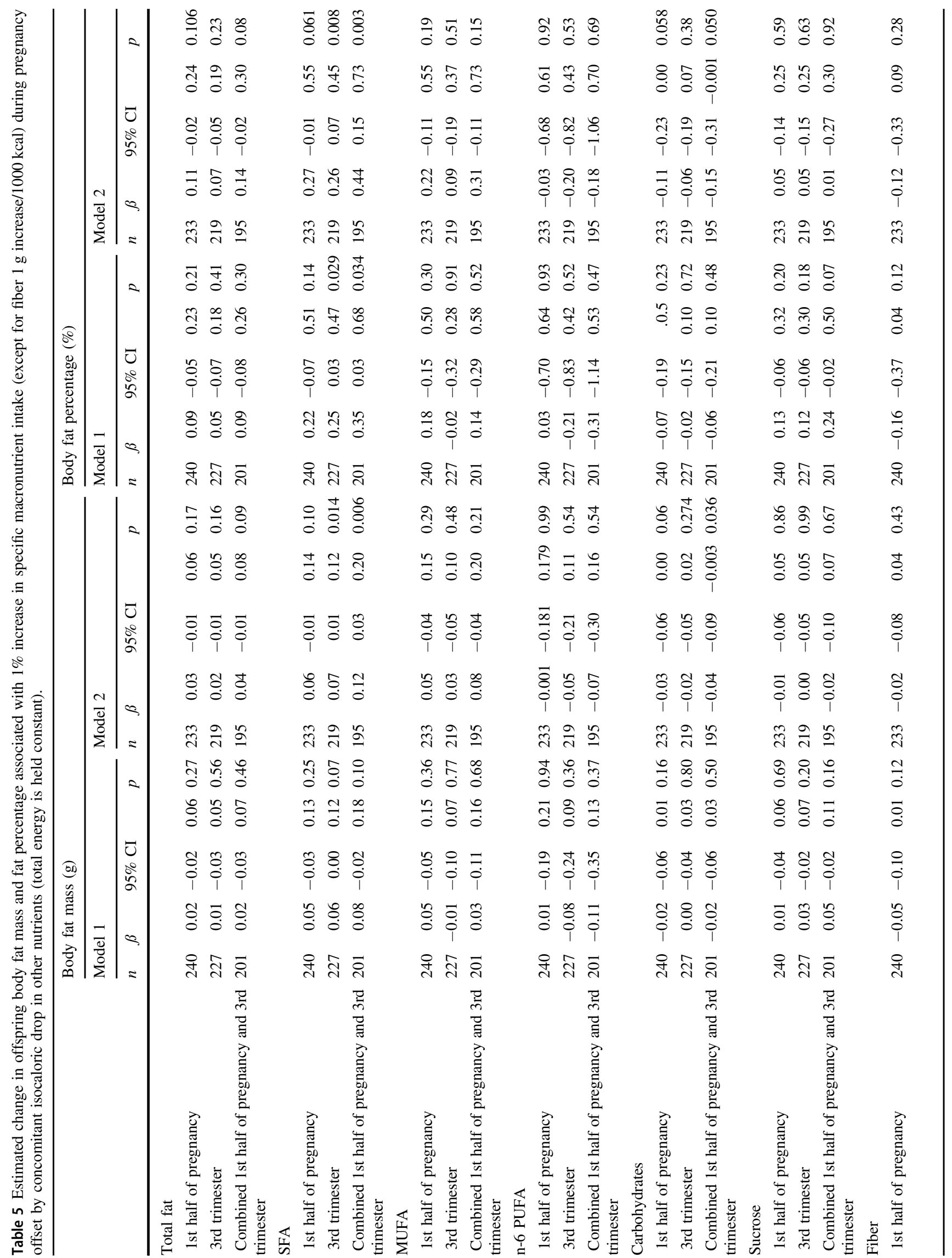




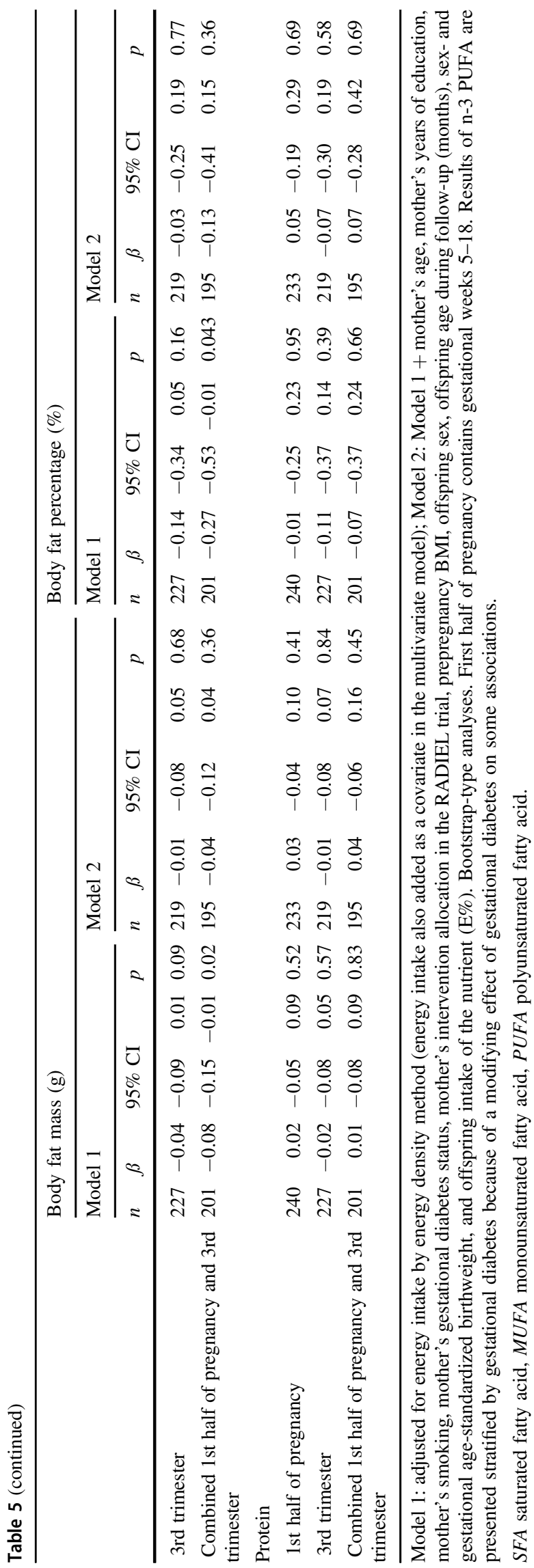

[17], maternal cumulative SFA intake during pregnancy was not associated with offspring BMI or overweight at 2-7 years [17]. Likewise, Brei et al. $(n=208)$ [8] did not find associations between SFA intake in early or late pregnancy and offspring weight, BMI, BFM, or subcutaneous or peritoneal fat area at birth or at the age of 1,3 , or 5 years. The participants in these studies were leaner and the proportion of mothers with GDM was null [17] or considerably lower [8], compared to the mothers of the present study. This may have contributed to the discrepancy in results, especially considering the differences in maternal, placental, and fetal lipid metabolism that characterize GDM/obese vs. non-GDM/non-obese pregnancies [28, 39]. Murrin et al. [16], on the other hand, discovered a positive association between maternal SFA intake in the first trimester and offspring weight at 5 years of age in 585 mother-child dyads. This result, if weight is interpreted as an indication of adiposity, is in line with our findings concerning third trimester SFA intake and combined SFA intake across gestation. It is possible that our smaller sample size may have diluted the association between the first half of pregnancy intake and offspring anthropometric indicators. Unfortunately, Murrin et al. did not report maternal background characteristics, such as BMI or GDM status, disallowing detailed comparisons [16]. Data from animal studies are consistent with our findings, since high maternal SFA intake has been shown to result in increased neonatal body mass [40], later adiposity [41], beta-cell loss and glucose intolerance [42], as well as impaired appetite regulation [43], in the offspring.

In the present study, maternal SFA intake during late pregnancy, as opposed to the first half of pregnancy, displayed a more pronounced association with offspring anthropometric indicators. This is logical since the last trimester of pregnancy is characterized by the most rapid fetal growth and fat accretion, which is further promoted by maternal obesity and/or diabetes which enhance the availability of lipids as a feto-placental fuel and trigger fetal hyperinsulinemia [28]. Nearly half of our cohort was composed of women with GDM, in whom maternal late pregnancy free fatty acid, glycerol and triacylglycerol concentrations have been shown to correlate with cord lipid concentrations, newborn size, and adiposity [44, 45]. An SFA-rich diet could also contribute to poorer maternal glycemic control via worsening of late-pregnancy insulin resistance [46], especially in our metabolically compromised study population. It is also possible that excess SFA could suppress the activity of specific enzymes $(\Delta 5$ and $\Delta 6)$ necessary for the synthesis of metabolically important longchain PUFAs in maternal and fetal tissues [47].

Brei et al. [8] found, in 186 mother-child dyads, that an incremental increase in maternal fat and protein intake at the expense of $\mathrm{CHO}$ in late but not in early pregnancy was 
associated with lower offspring BFM up to 5 years. This is in contrast to our study in which higher maternal $\mathrm{CHO}$ intake was associated with lower offspring BFM and BF\% at 5 years of age. However, the average $\mathrm{CHO}$ intake was $\sim 7 \mathrm{E} \%$ units higher in the study population of Brei et al. as compared to our study population, which could explain the discrepancy in our results. The finding that only cumulative $\mathrm{CHO}$ intake across pregnancy was associated with $\mathrm{BFM}$ and $\mathrm{BF} \%$ probably attributes to the higher accuracy of measurement achieved with 6 days rather than 3 days of food records.

We did not find an association between maternal macronutrient intake and newborn relative birthweight, in agreement with some studies [41] but in contradiction to others [48]. Our results may reflect the fact that birthweight is not a measure of adiposity. It is possible that the children with the highest BFM/BF\% values already had higher adiposity at birth [49], but it is also plausible that fetal programming predisposed them to increased fat accumulation after the neonatal period. The latter speculation is supported by a meta-analysis of studies in rodents, which concluded that maternal high-fat (mostly saturated) intake did not affect offspring birthweight but increased weaning weight, final bodyweight, and adiposity [41]. This is in agreement with our results showing no association between maternal SFA intake and offspring birthweight, but positive associations between maternal SFA intake and offspring $\mathrm{BFM}$ and $\mathrm{BF} \%$ at 5 years of age. The possible cause-effect relationships between maternal SFA intake and offspring metabolism are myriad and remain to be elucidated, with potential mechanisms ranging from adverse impacts of SFA on feto-placental mitochondrial metabolism to alterations of maternal/fetal gut microbiota [50-52].

A strength of our study is the availability of direct measurement of offspring adiposity as BFM and BF\%, instead of relying only on bodyweight, BMI, or WHtR [53]. We were able to adjust for a wide range of variables, in order to minimize residual confounding. Since all the women who participated in the present study were either obese or had a history of GDM, and consequently their fetuses were exposed to an altered intrauterine environment, the results cannot be generalized to metabolically healthy pregnancies. Our relatively small sample size is another limitation, which may have attenuated some of the observed associations between maternal macronutrient intakes and offspring anthropometric indicators. Due to lack of data on newborn $\mathrm{BFM}, \mathrm{BF} \%$, or metabolic parameters (e.g., insulin or Cpeptide levels), we were not able to assess whether associations between specific macronutrient intakes and offspring adiposity already existed in infancy. Similarly, due to lack of data, we could not adjust for maternal macronutrient intake during lactation as a possible confounder.

In metabolically burdened women, associations between the first half of pregnancy n-3 PUFA intake and offspring
BFM and $\mathrm{BF} \%$ at 5 years of age were modified by GDM. In the first half of pregnancy, higher n-3 PUFA intake was associated with lower offspring $\mathrm{BFM}$ and $\mathrm{BF} \%$ in women with normal glucose tolerance during pregnancy and higher offspring BFM and BF\% in women with GDM. Higher intake of SFA during pregnancy was associated with higher ISOBMI, BFM, and BF\% in the offspring at 5 years of age, whereas higher intake of CHOs was associated with lower offspring BFM and BF\%. This study introduces novel evidence of possible fetal programming by maternal macronutrient intake in metabolically burdened women who constitute a growing proportion of obstetric populations worldwide. Taken together, our findings add to the accumulating evidence on the role of maternal macronutrient intake in offspring metabolic programming and suggest that its effects may vary between normoglycemic women and women with GDM and depend on the timing of feto-placental exposure.

\section{Data availability}

The dataset supporting the conclusions of this article is available upon a reasonable request from the authors.

\section{Code availability}

The code supporting the conclusions of this article is available upon a reasonable request from the authors.

Acknowledgements We thank Antti Saari for providing formulas based on Finnish growth references for children for calculating sexspecific BMI-for-age (ISO-BMI). The work was funded by the Ahokas Foundation, Finnish Foundation for Cardiovascular Disease, Special State Subsidy for Health Science Research of Helsinki University Central Hospital, Samfundet Folkhälsan, Finnish Diabetes Research Foundation, Foundation for Medical Research Liv och Hälsa, Kulturfonden, Juho Vainio Foundation, Maud Kuistila Memorial Foundation, Finnish Cultural Foundation, Biomedicum Helsinki Research Foundation, Finnish Medical Foundation, Viipuri Tuberculosis Foundation, State Provincial Office of Southern Finland, and the Social Insurance Institution of Finland. The funders had no role in the design, implementation, analysis, or interpretation of the data.

Author contributions The authors' contributions were as follows: JM, MMK, BS-L, EH, EE, SA, and SK designed the research; MMK, SK, BS-L, and EH conducted the research; JM analyzed the data; JM and MMK wrote the paper; BS-L, EH, EE, SA, and SK critically revised the manuscript with expert advice and comments; JM had primary responsibility for final content; all authors read and approved the final manuscript.

Funding Open Access funding provided by University of Helsinki including Helsinki University Central Hospital.

\section{Compliance with ethical standards}

Conflict of interest The authors declare that they have no conflict of interest. 
Publisher's note Springer Nature remains neutral with regard to jurisdictional claims in published maps and institutional affiliations.

Open Access This article is licensed under a Creative Commons Attribution 4.0 International License, which permits use, sharing, adaptation, distribution and reproduction in any medium or format, as long as you give appropriate credit to the original author(s) and the source, provide a link to the Creative Commons license, and indicate if changes were made. The images or other third party material in this article are included in the article's Creative Commons license, unless indicated otherwise in a credit line to the material. If material is not included in the article's Creative Commons license and your intended use is not permitted by statutory regulation or exceeds the permitted use, you will need to obtain permission directly from the copyright holder. To view a copy of this license, visit http://creativecommons. org/licenses/by/4.0/.

\section{References}

1. WHO. Facts and figures on childhood obesity. 2020. http://www. who.int/end-childhood-obesity/facts/en/. Accessed 1 Feb 2020.

2. Mäki P, Lehtinen-Jacks S, Vuorela N, Levälahti E, Koskela T, Saari A, et al. Register of Primary Health Care Visits (Avohilmo) as data source for monitoring the prevalence of overweight among Finnish children. Finnish Med J. 2017;72:209-15.

3. Buttitta M, Iliescu C, Rousseau A, Guerrien A. Quality of life in overweight and obese children and adolescents: a literature review. Qual Life Res. 2014;23:1117-39.

4. Di Angelantonio E, Bhupathiraju S, Wormser D, Gao P, Kaptoge $\mathrm{S}$, Berrington de Gonzalez A, et al. Body-mass index and all-cause mortality: individual-participant-data meta-analysis of 239 prospective studies in four continents. Lancet. 2016;388:776-86.

5. Flegal KM, Kit BK, Orpana H, Graubard BI. Association of allcause mortality with overweight and obesity using standard body mass index categories: a systematic review and meta-analysis. JAMA. 2013;309:71-82.

6. Magalhães EI, da Silva, Lima NP, Menezes AMB, Gonçalves H, Wehrmeister FC, et al. Maternal smoking during pregnancy and offspring body composition in adulthood: results from two birth cohort studies. BMJ Open. 2019;9:e23852.

7. Mourtakos SP, Tambalis KD, Panagiotakos DB, Antonogeorgos G, Arnaoutis G, Karteroliotis K, et al. Maternal lifestyle characteristics during pregnancy, and the risk of obesity in the offspring: a study of 5,125 children. BMC Pregnancy Childbirth. 2015; 15:66-Z

8. Brei C, Stecher L, Meyer DM, Young V, Much D, Brunner S, et al. Impact of dietary macronutrient intake during early and late gestation on offspring body composition at birth, 1,3 , and 5 years of age. Nutrients. 2018;10:579.

9. Kaseva N, Vääräsmäki $M$, Matinolli $H$, Sipola-Leppänen $M$, Tikanmäki M, Heinonen K, et al. Pre-pregnancy overweight or obesity and gestational diabetes as predictors of body composition in offspring twenty years later: evidence from two birth cohort studies. Int J Obes. 2018;42:872-9.

10. Godfrey KM, Reynolds RM, Prescott SL, Nyirenda M, Jaddoe VWV, Eriksson JG, et al. Influence of maternal obesity on the long-term health of offspring. Lancet Diabetes Endocrinol. 2017;5:53-64.

11. Hammoud NM, Visser GHA, van Rossem L, Biesma DH, Wit JM, de Valk HW. Long-term BMI and growth profiles in offspring of women with gestational diabetes. Diabetologia. 2018;61: 1037-45.

12. Chia A, Tint M, Han CY, Chen L, Colega M, Aris IM, et al. Adherence to a healthy eating index for pregnant women is associated with lower neonatal adiposity in a multiethnic Asian cohort: the Growing Up in Singapore Towards healthy Outcomes (GUSTO) Study. Am J Clin Nutr. 2018;107:71-9.

13. Shapiro ALB, Kaar JL, Crume TL, Starling AP, Siega-Riz AM, Ringham BM, et al. Maternal diet quality in pregnancy and neonatal adiposity: the Healthy Start Study. Int J Obes. 2016;40: 1056-62.

14. Crume TL, Brinton JT, Shapiro A, Kaar J, Glueck DH, Siega-Riz $\mathrm{AM}$, et al. Maternal dietary intake during pregnancy and offspring body composition: the Healthy Start Study. Am J Obstet Gynecol. 2016;215:609.e1-8.

15. Blumfield ML, Hure AJ, MacDonald-Wicks LK, Smith R, Simpson SJ, Giles WB, et al. Dietary balance during pregnancy is associated with fetal adiposity and fat distribution. Am J Clin Nutr. 2012;96:1032-41.

16. Murrin C, Shrivastava A, Kelleher CC. Maternal macronutrient intake during pregnancy and 5 years postpartum and associations with child weight status aged five. Eur $\mathrm{J}$ Clin Nutr. 2013;67:670-9.

17. Hakola L, Takkinen H, Niinistö S, Ahonen S, Erlund I, Rautanen $\mathrm{J}$, et al. Maternal fatty acid intake during pregnancy and the development of childhood overweight: a birth cohort study. Pediatr Obes. 2017;12:26-37.

18. Donahue SM, Rifas-Shiman SL, Gold DR, Jouni ZE, Gillman MW, Oken E. Prenatal fatty acid status and child adiposity at age 3 y: results from a US pregnancy cohort. Am J Clin Nutr. 2011;93:780-8.

19. Maslova E, Rytter D, Bech BH, Henriksen TB, Olsen SF, Halldorsson TI. Maternal intake of fat in pregnancy and offspring metabolic health - a prospective study with 20 years of follow-up. Clin Nutr. 2016;35:475-83.

20. Rono K, Grotenfelt NE, Klemetti MM, Stach-Lempinen B, Huvinen E, Meinila $J$, et al. Effect of a lifestyle intervention during pregnancy-findings from the Finnish gestational diabetes prevention trial (RADIEL). J Perinatol. 2018;38:1157-64.

21. Sääksjärvi K, Reinivuo H. Food portion sizes. Helsinki: National Institute of Health and Welfare; 2004.

22. Pihkala J, Hakala T, Voutilainen P, Raivio K. [Characteristic of recent fetal growth curves in Finland]. Duodecim. 1989;105: 1540-6.

23. Saari A, Sankilampi U, Hannila M, Kiviniemi V, Kesseli K, Dunkel L. New Finnish growth references for children and adolescents aged 0 to 20 years: length/height-for-age, weightfor-length/height, and body mass index-for-age. Ann Med. 2011;43:235-48.

24. Rudolph MC, Jackman MR, Presby DM, Houck JA, Webb PG, Johnson GC, et al. Low neonatal plasma n-6/n-3 PUFA ratios regulate offspring adipogenic potential and condition adult obesity resistance. Diabetes. 2018;67:651-61.

25. Voortman T, Tielemans MJ, Stroobant W, Schoufour JD, Kiefte-de Jong JC, Steenweg-de Graaff J, et al. Plasma fatty acid patterns during pregnancy and child's growth, body composition, and cardiometabolic health: the Generation R Study. Clin Nutr. 2018;37:984-92.

26. Vahdaninia M, Vahdaninia M, Mackenzie H, Mackenzie H, Dean $\mathrm{T}$, Dean $\mathrm{T}$, et al. The effectiveness of $\omega-3$ polyunsaturated fatty acid interventions during pregnancy on obesity measures in the offspring: an up-to-date systematic review and meta-analysis. Eur J Nutr. 2019;58:2597-613.

27. Hanebutt FL, Demmelmair H, Schiessl B, Larque E, Koletzko B. Long-chain polyunsaturated fatty acid (LC-PUFA) transfer across the placenta. Clin Nutr. 2008;27:685-93.

28. Herrera E, Desoye G. Maternal and fetal lipid metabolism under normal and gestational diabetic conditions. Horm Mol Biol Clin Investig. 2016;26:109-27.

29. Pagán A, Prieto-Sánchez MT, Blanco-Carnero JE, Gil-Sánchez A, Parrilla JJ, Demmelmair $\mathrm{H}$, et al. Materno-fetal transfer of 
docosahexaenoic acid is impaired by gestational diabetes mellitus. Am J Physiol Endocrinol Metab. 2013;305:826.

30. Prieto-Sánchez MT, Ruiz-Palacios M, Blanco-Carnero JE, Pagan A, Hellmuth C, Uhl O, et al. Placental MFSD2a transporter is related to decreased DHA in cord blood of women with treated gestational diabetes. Clin Nutr. 2017;36:513-21.

31. Shokry E, Marchioro L, Uhl O, Bermúdez MG, García-Santos JA, Segura MT, et al. Transgenerational cycle of obesity and diabetes: investigating possible metabolic precursors in cord blood from the PREOBE study. Acta Diabetol. 2019;56:1073-82.

32. Fattuoni C, Mandò C, Palmas F, Anelli GM, Novielli C, Parejo Laudicina E, et al. Preliminary metabolomics analysis of placenta in maternal obesity. Placenta. 2018;61:89-95.

33. Jokelainen $M$, Stach-Lempinen $B$, Rönö $K$, Nenonen A, Kautiainen H, Teramo K, et al. Oral glucose tolerance test results in early pregnancy: a Finnish population-based cohort study. Diabetes Res Clin Pract. 2020;162:108077.

34. Catalano PM. Trying to understand gestational diabetes. Diabetic Med. 2014;31:273-81.

35. Abbade J, Klemetti MM, Farrell A, Ermini L, Gillmore T, Sallais $\mathrm{J}$, et al. Increased placental mitochondrial fusion in gestational diabetes mellitus: an adaptive mechanism to optimize fetoplacental metabolic homeostasis? BMJ Open Diabetes Res Care. 2020;8:e00923.

36. Desoye G, van Poppel M. The feto-placental dialogue and diabesity. Best Pract Res Clin Obstet Gynaecol. 2015;29:15-23.

37. Lassance L, Haghiac M, Leahy P, Basu S, Minium J, Zhou J, et al. Identification of early transcriptome signatures in placenta exposed to insulin and obesity. Am J Obstet Gynecol. 2015;212: 647.e1-11.

38. O'Tierney-Ginn P, Presley L, Myers S, Catalano P. Placental growth response to maternal insulin in early pregnancy. J Clin Endocrinol Metab. 2015;100:159-65.

39. Stirm L, Kovárová M, Perschbacher S, Michlmaier R, Fritsche L, Siegel-Axel D, et al. BMI-independent effects of gestational diabetes on human placenta. J Clin Endocrinol Metab. 2018;103: 3299-309.

40. Strakovsky RS, Zhang X, Zhou D, Pan Y. Gestational high fat diet programs hepatic phosphoenolpyruvate carboxykinase gene expression and histone modification in neonatal offspring rats. $\mathbf{J}$ Physiol. 2011;589:2707-17.

41. Ribaroff GA, Wastnedge E, Drake AJ, Sharpe RM, Chambers TJG. Animal models of maternal high fat diet exposure and effects on metabolism in offspring: a meta-regression analysis. Obes Rev. 2017;18:673-86.

42. Siemelink M, Verhoef A, Dormans JAMA, Span PN, Piersma AH. Dietary fatty acid composition during pregnancy and lactation in the rat programs growth and glucose metabolism in the offspring. Diabetologia. 2002;45:1397-403.

43. Rother E, Kuschewski R, Alcazar MAA, Oberthuer A, Bae-Gartz I, Vohlen C, et al. Hypothalamic JNK1 and IKK $\beta$ activation and impaired early postnatal glucose metabolism after maternal perinatal high-fat feeding. Endocrinology. 2012;153:770-81.

44. Schaefer-Graf UM, Graf K, Kulbacka I, Kjos SL, Dudenhausen J, Vetter K, et al. Maternal lipids as strong determinants of fetal environment and growth in pregnancies with gestational diabetes mellitus. Diabetes Care. 2008;31:1858-63.

45. Schaefer-Graf UM, Meitzner K, Ortega-Senovilla H, Graf K, Vetter K, Abou-Dakn M, et al. Differences in the implications of maternal lipids on fetal metabolism and growth between gestational diabetes mellitus and control pregnancies. Diabet Med. 2011;28:1053-9.

46. Yki-Järvinen H. Nutritional modulation of non-alcoholic fatty liver disease and insulin resistance. Nutrients. 2015;7:9127-38.

47. Das UN. Is metabolic syndrome $X$ a disorder of the brain with the initiation of low-grade systemic inflammatory events during the perinatal period? J Nutr Biochem. 2007;18:701-13.

48. Sharma SS, Greenwood DC, Simpson NAB, Cade JE. Is dietary macronutrient composition during pregnancy associated with offspring birth weight? An observational study. British J Nutr. 2018;119:330-9.

49. Catalano PM, Farrell K, Thomas A, Huston-Presley L, Mencin P, de Mouzon SH, et al. Perinatal risk factors for childhood obesity and metabolic dysregulation. Am J Clin Nutr. 2009;90:1303-13.

50. Wolters M, Ahrens J, Romaní-Pérez M, Watkins C, Sanz Y, Benítez-Páez A, et al. Dietary fat, the gut microbiota, and metabolic health-a systematic review conducted within the MyNewGut project. Clin Nutr. 2019;38:2504-20.

51. Putti R, Sica R, Migliaccio V, Lionetti L. Diet impact on mitochondrial bioenergetics and dynamics. Front Physiol. 2015;6:109.

52. Isganaitis E. Developmental programming of body composition: update on evidence and mechanisms. Curr Diab Rep. 2019;19:60.

53. Jensen SM, Mølgaard C, Ejlerskov KT, Christensen LB, Michaelsen $\mathrm{KF}$, Briend A. Validity of anthropometric measurements to assess body composition, including muscle mass, in 3-year-old children from the SKOT cohort. Matern Child Nutr. 2015;11:398-408. 